\title{
Influence of wave dispersion on the self-consistent dynamics of cyclotron-laser-type systems
}

\author{
R. Pakter, R. S. Schneider, and F. B. Rizzato \\ Instituto de Física, Universidade Federal do Rio Grande do Sul, Caixa Postal 15051, 91501-970 Porto Alegre, \\ Rio Grande do Sul, Brazil
}

(Received 23 October 1992)

\begin{abstract}
In this work, we make use of a Hamiltonian formalism to analyze the wave-particle dynamical interaction that takes place in cyclotronic systems. It is shown that the usual model of test particles moving in externally given wave fields is good only when the amplitude of the radiation is large enough; otherwise, wave dynamics must be considered and the dynamics mentioned undergoes some considerable changes. In this regard, the appearance of new fixed points and the saturation of the autoresonance process are both analyzed as functions of the dispersion relation of the laser field.
\end{abstract}

PACS number(s): 41.75.Ht, 41.60.-m

With the advent of radiation-generating systems such as free-electron lasers, cyclotron autoresonance masers, gyrotrons, ion-channel lasers, and others, a good deal of effort has been directed toward the analysis of the interaction of relativistic particles and large-amplitude electromagnetic waves. In many cases, quantities of interest such as energy gain are usually obtained by considering the electronic dynamics in given external wave fields. This kind of approximation can be fairly justified if one considers either waves with large enough amplitudes or beams with very tenuous densities, so that particles do not appreciably react back on the electromagnetic fluctuations. On the other hand, when these conditions are not fulfilled, a self-consistent analysis is necessary where particles and fields are to be simultaneously treated as a many-body dynamical system. In fact, this self-consistent analysis has been carried out analytically $[1,2]$ and numerically [3-7], both for amplifier and accelerator configurations. Among other results, it has been shown how the finite amount of free electromagnetic energy may act as a limiter for wave-particle energy exchanges.

Besides wave dynamics, one should also consider dispersive effects connected with the electromagnetic modes [5]. This appears to be the correct assumption because of two interrelated aspects: (i) in usual physical configurations, such as that of wave-guided systems, wave propagation is indeed dispersive and (ii) dispersion detunes the relative wave-particle phases, thereby becoming another important factor in limiting the above-mentioned energy-exchange processes.

With those points in mind, our purpose in the present work is to investigate a cyclotron-laser-type system for which both the finite amount of electromagnetic energy and the dispersive characteristics of the electromagnetic beam are simultaneously taken into consideration. We intend to focus our attention on accelerator configurations, highlighting the analysis of the autoresonance process and the location of fixed points for the overall dy- namics. In order to do that, we shall make use of a canonical technique where all the relevant equations for both particles and wave may be derived from one generalized Hamiltonian [2].

As a model, let us consider an electron beam and a circularly polarized electromagnetic wave, copropagating along the homogeneously magnetized $z$ axis of the chosen reference frame.

With the circularly polarized wave-vector potential written as

$$
\frac{e}{m c^{2}} \mathbf{A} \equiv-\frac{1}{2} \sqrt{\rho} e^{i \sigma} e^{i(f z-t)} \hat{\mathbf{e}}_{c}+\text { c.c. },
$$

where $\rho$ and $\sigma$ have a slow time dependence, $e$ is the electron charge, $\hat{\mathbf{e}}_{c} \equiv \hat{\mathbf{x}}+i \hat{\mathbf{y}}$, time and space have been normalized to $\omega$ and $\omega / c$ with $\omega$ and $k$ as the wave frequency and wave vector, respectively, and dispersive properties of the wave are modeled in the generic form $\omega /(c k)=\sqrt{1+g^{2}} \equiv f^{-1}$, with $g$ as a factor accounting for dispersion (it could be a factor connected with the finite transverse dimensions of some guiding system), it becomes possible to cast the Hamiltonian that governs the electron dynamics in the form

$$
\begin{aligned}
\mathcal{H}= & \left\{1+\left[P_{x}+\sqrt{\rho} \cos (f z-t+\sigma)\right]^{2}\right. \\
& \left.+\left[x+P_{y}-\sqrt{\rho} \sin (f z-t+\sigma)\right]^{2}+P_{z}^{2}\right\}^{1 / 2}
\end{aligned}
$$

with $\mathcal{H} / m c^{2} \rightarrow \mathcal{H}$ and $\mathbf{P} / m c \rightarrow \mathbf{P}$. Denoting $B_{z, 0}$ as the background magnetic field, we assume the ressonance condition $\omega=k_{z} v_{0 z}+|e| B_{z, 0} / m c \gamma_{0}$ to be operating at least during the initial stages; besides we assume accelerating particles with small values of the initial longitudinal velocity $v_{z 0}$ and $\gamma_{0} \approx 1$.

The structure of the Hamiltonian can be made simpler if one introduces canonical guiding-center variables. In order to do that in the most convenient way, let us first rewrite the Hamiltonian (2) as a power series of the small quantity $\sqrt{\rho}$, considering only the leading-order contribution:

$$
\mathcal{H}=\Gamma+\frac{\sqrt{\rho}\left[P_{x} \cos (f z-t+\sigma)-\left(x+P_{y}\right) \sin (f z-t+\sigma)\right]}{\Gamma}
$$


with

$$
\Gamma \equiv \sqrt{1+P_{x}^{2}+\left(x+P_{y}\right)^{2}+P_{z}^{2}}
$$

Then, the following generating function:

$$
\mathcal{F}=P_{y} P_{x}-\frac{P_{x}^{2}}{2} \operatorname{tg} x^{\prime}-y^{\prime} P_{y}-z^{\prime} P_{z},
$$

with primes designating new variables, enables one to write [5] $P_{x}=\sqrt{2 I} \cos \phi$ and $x+P_{y}=\sqrt{2 I} \sin \phi$, with $I \equiv P_{x}^{\prime}$ and $\phi \equiv x^{\prime}$. With that, on dropping the primes wherever they appear and making use of the additional canonical transformation [5] $\phi+f z-t \rightarrow \phi, P_{z} \rightarrow P_{z}+f I$, and $\mathcal{H} \rightarrow \mathcal{H}+I$, the Hamiltonian (3) can be rewritten as

$$
\mathcal{H}=-I+\Gamma+\frac{\sqrt{\rho} \sqrt{2 I}}{\Gamma} \cos (\phi+\sigma),
$$

where now one shall consider

$$
\Gamma=\sqrt{1+2 I+\left(P_{z}+f I\right)^{2}} .
$$

Were our interest to study the dynamics of electrons in given electromagnetic fields, we could stop with the calculations at the present stage. However, as said before, we wish to understand what happens when the dynamics of the fluctuating wave field is taken into consideration. In order to do that one has to derive the equations for the fields and then to analyze the whole set of dynamical laws on the same basis. So, let us proceed to see what can be done with those fields and how to derive all the relevant dynamical equations from a generalized Hamiltonian principle.

The slow-time dimensional dynamics for the complex amplitude of the electromagnetic field is readily derived from Maxwel's equation for the vector potential $\mathbf{A}$ as

$$
i \omega d_{t}\left(\sqrt{\rho} e^{i \sigma}\right)=\frac{2 \pi e}{m c V T} \int \mathbf{J}_{\perp} \cdot \hat{\mathbf{e}}_{c}^{*} e^{-i\left(k z^{\prime}-\omega t^{\prime}\right)} d^{3} \mathbf{r}^{\prime} d t^{\prime},
$$

where we have performed an inverse discrete Fourier transform over the fast (primed) variables, introducing the volume $V$, the period $T$, and $d_{t} \equiv d / d t$. The quantity $\mathbf{J}_{\perp}$ is the current density on the $(x, y)$ plane, written as

$$
\mathbf{J}_{\perp}=\sum_{i} e \mathbf{v}_{\perp, i}\left(t^{\prime}\right) \delta\left(\mathbf{r}^{\prime}-\mathbf{r}_{i}\left(t^{\prime}\right)\right)
$$

with the subscripts $i$ labeling the particles present in the system, $\mathbf{v}_{\perp, i}\left(t^{\prime}\right)$ denoting the perpendicular (to the $z$ axis) component of their velocities, and $\mathbf{r}_{i}\left(t^{\prime}\right)$ representing their instantaneous positions.

Now, on writing the velocity $\mathbf{v}_{\perp, i}$ in terms of the nondimensional canonical variables and assuming again the densities to be so low that dielectric effects can be disregarded, it becomes possible to cast Eq. (7) in the form

$$
-i d_{t}\left(\sqrt{\rho} e^{i \sigma}\right)=-\frac{2 \pi e^{2}}{m \omega^{2} V} \sum_{i} \frac{\sqrt{2 I_{i}}}{\Gamma_{i}} e^{-i\left[\phi_{i}(t)+z_{i}(t)-t\right]}
$$

where the resonant wave-particle phase $\phi_{i}+z_{i}-t$ is a variable depending only on the slow time scales.

In order to perform the summation indicated in the above equation, one makes use of the so-called macroparticle approximation. It is known that phase bunching occurs in this kind of system in conjunction with wave amplification $[5,8]$, and the macroparticle approximation goes further in the sense that it assumes an extreme bunching condition for which the wave-particle relative phase $\phi_{i}+z_{i}-t$ is the same for all particles [1,2,5,8-10]. With that, one can write for the wave field

$$
-i d_{t}\left(\sqrt{\rho} e^{i \sigma}\right)=-\omega_{p}^{2} \frac{\sqrt{2 I}}{2 \Gamma} e^{-i[\phi+f z(t)-t]}
$$

where

$$
\omega_{p}^{2} \equiv \frac{4 \pi e^{2} N}{V m \omega^{2}},
$$

with $N$ as the total number of particles present in the system. Splitting Eq. (10) into real and imaginary parts and noticing that the factor $\phi+f z(t)-t$ is to be considered as the new canonical $\phi$ coordinate leads to

$$
d_{t} \rho=-2 \omega_{p}^{2} \frac{\sqrt{2 I}}{2 \Gamma} \sqrt{\rho} \sin (\phi+\sigma)
$$

and

$$
d_{t} \sigma=-\omega_{p}^{2} \frac{\sqrt{2 I}}{2 \Gamma} \frac{1}{\sqrt{\rho}} \cos (\phi+\sigma) .
$$

Now, if one rescales $\rho$ according to

$$
\rho=\lambda \rho^{\prime}, \quad \lambda \equiv 2 \omega_{p}^{2}
$$

the interesting and final conclusion is that all the relevant dynamical equations for both particles and fields can be derived from one generalized Hamiltonian given by

$$
\mathcal{H}=-I+\Gamma+\frac{\sqrt{\lambda \rho^{\prime}} \sqrt{2 I}}{2 \Gamma} \cos \left(\phi-\sigma^{\prime}\right)
$$

where $\rho^{\prime}$ is the "momentum" corresponding to the wave field and $\sigma^{\prime}(=-\sigma)$ is the canonically conjugated coordinate. If one drops the primes once again, the following canonical transformation:

$$
\phi-\sigma \rightarrow \phi, \quad \rho \rightarrow \rho-I,
$$

reduces the degrees of freedom $[\rho$ is now a constant of motion that in virtue of the scaling relations below Eqs. (12) and (13) can be thought of as the initial electromagnetic energy content available per particle; it is a normalized energy density divided by particle density] and allows us to write a final effective canonical system as

$$
\begin{gathered}
\mathcal{H}=-I+\Gamma+\frac{\sqrt{\lambda(\rho-I)} \sqrt{2 I}}{2 \Gamma} \cos \phi, \\
\dot{I}=-\frac{\partial \mathcal{H}}{\partial \phi},
\end{gathered}
$$




$$
\ddot{\phi}=\frac{\partial \mathcal{H}}{\partial I} \text {. }
$$

Let us first investigate the influence of wave dynamics and wave dispersion on the autoresonance process. In order to do that, we recall that the autoresonance process takes place when we set $f=1, \rho-I \rightarrow \rho$, and $P_{z}=0$ in Eq. (15) with $\phi(t=0)=(2 m+1 / 2) \pi$, which means an approximation of large-amplitude dispersionless laser waves, energizing both the transverse and longitudinal particle dynamics (observe that, although the canonical transformed $P_{z}$ is zero, the physical $P_{z}$ grows proportionally to $I)$. Under such circumstances, the canonical equations for $I$ and $\phi$ imply the constancy of the angular variable and the unlimited growth of the momentum $I$. On the other hand, when wave dynamics is taken into account with $f=1$ and the previous initial condition for $\phi$, the canonical equations indicate that the energization process proceeds without variations of $\phi(t)$, up to a maximum value for $I$, readily evaluated as $I_{\max }=\rho$, where it can be shown that the initial electromagnetic energy is totally converted into the particle energy $\Gamma$. To evaluate $I_{\max }$ in the generic situation $f<1$, it is convenient to combine our canonical equations (15)-(17) in order to obtain a closed equation for $I$ in the form

$$
(\dot{I})^{2}+V(I)=0 .
$$

In the equation above, $V(I)$ is an effective potential driving $I$ excursions. It is written as

$$
V(I)=-\frac{\lambda I(\rho-I)}{2 \Gamma^{2}}+(h+I-\Gamma)^{2},
$$

with $h$ as the numerical (and constant) value of $\mathcal{H}$. The quantity $I_{\max }$ shall be sought for as the first zero of the function $V(I)$. In Fig. 1 we plot $I_{\max }$ vs $f$ in the case where wave dynamics is not included [curve $(a)$ ] and the case where it is [curve $(b)$ ]. It is seen that wave dynamics reduces the numerical value of $I_{\max }$ for all $f$ 's; this reduction is even dramatic when one gets close to $f=1$ where, as said before, wave dynamics limits an otherwise unbounded growth of $I$. Apart from that, one may observe that the larger the factor $\rho$, the stronger the tendency of curves $(a)$ and $(b)$ to stay together; in fully relativistic situations where $\rho \geq 1$ (interesting to note is that the momentum $I$ is bounded by $\rho$ and not by the small "physical" field $\lambda \rho$; therefore it can become a fully relativistic variable), any noticeable differences between curves $(a)$ and $(b)$ come just from that region extremely close to $f=1$.

As for the topology on the phase space, let us focus our attention on the behavior of the fixed points for the considered dynamics. These fixed points are obtained as the solutions of the coupled set

$$
\begin{gathered}
\dot{I}_{\mathrm{FP}}=-\frac{\partial \mathcal{H}}{\partial \phi}\left(I_{\mathrm{FP}}, \phi_{\mathrm{FP}}\right)=0 \\
\dot{\phi}_{\mathrm{FP}}=\frac{\partial \mathcal{H}}{\partial I}\left(I_{\mathrm{FP}}, \phi_{\mathrm{FP}}\right)=0 .
\end{gathered}
$$

From the first of these equations, Eq. (20), one finds out
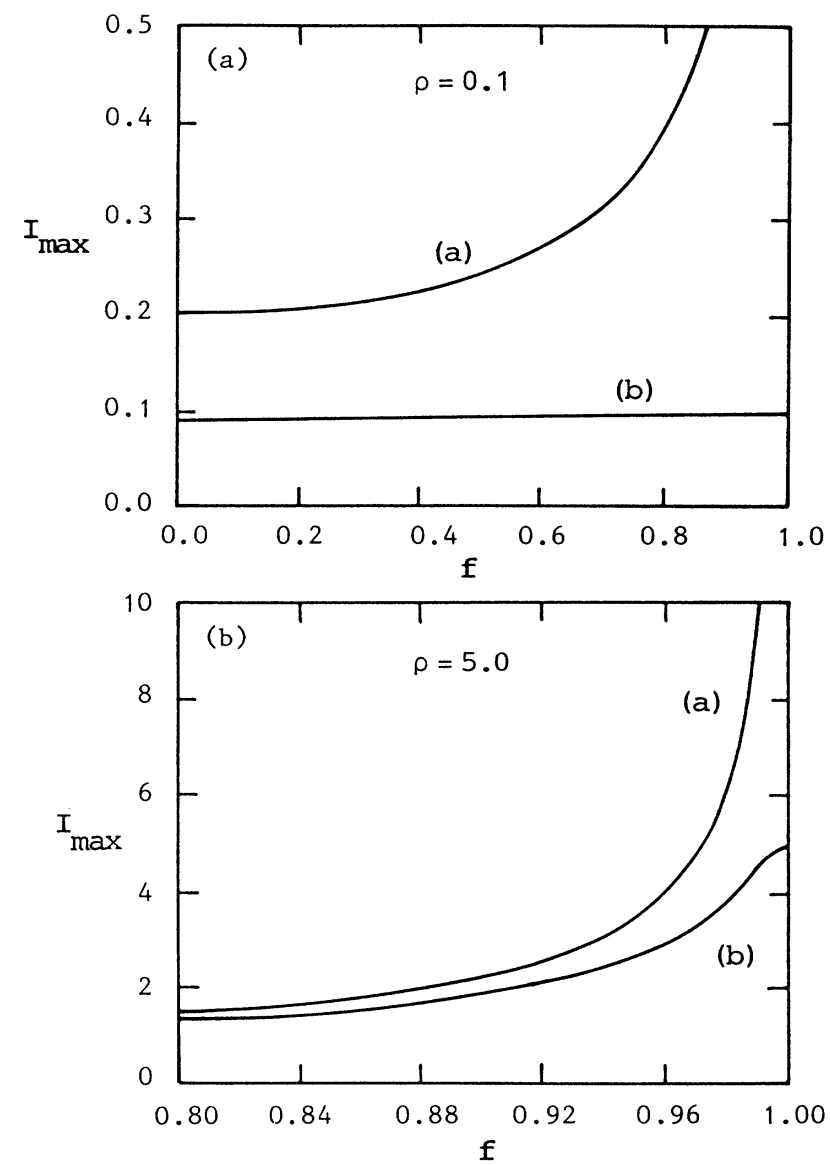

FIG. 1. $I_{\max }$ vs $f$ for (a) $\lambda=0.01, h=1.0, \rho=0.1$ and (b) $\rho=5.0$. In curve (a) wave dynamics is not included and in $(b)$ it is.

that at these points, the phase angles are such that

$$
\cos \left(\phi_{\mathrm{FP}}\right) \equiv S= \pm 1
$$

and from the second, Eq. (21), one is able to calculate

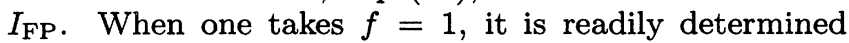
that both classes of fixed points, the one corresponding to $S=+1$ and the one corresponding to $S=-1$, occur at a same value along the $I$ axis, $I_{\mathrm{FP}}=\rho /(2+\rho)$. However, in the case of arbitrary values of $f$, each class has its own $I_{\mathrm{FP}}$. A good approximation indicates that as we take $f \rightarrow 0$ one gets

$$
I_{\mathrm{FP}}(S=+1) \rightarrow \frac{2}{3} \frac{\rho}{1+2 \rho}
$$

and

$$
I_{\mathrm{FP}}(S=-1) \rightarrow \rho\left(1-\frac{\lambda}{8} \frac{1}{(\sqrt{1+2 \rho}-1)^{2}}\right) .
$$

From these equations, it is not difficult to see that while the $S=+1$ fixed points are displaced toward lower values of $I$ for $f<1$, the $S=-1$ ones come to a situation where $I_{\mathrm{FP}} \approx \rho$. In Figs. $2(\mathrm{a})$ and $2(\mathrm{~b})$ we plot the tra- 

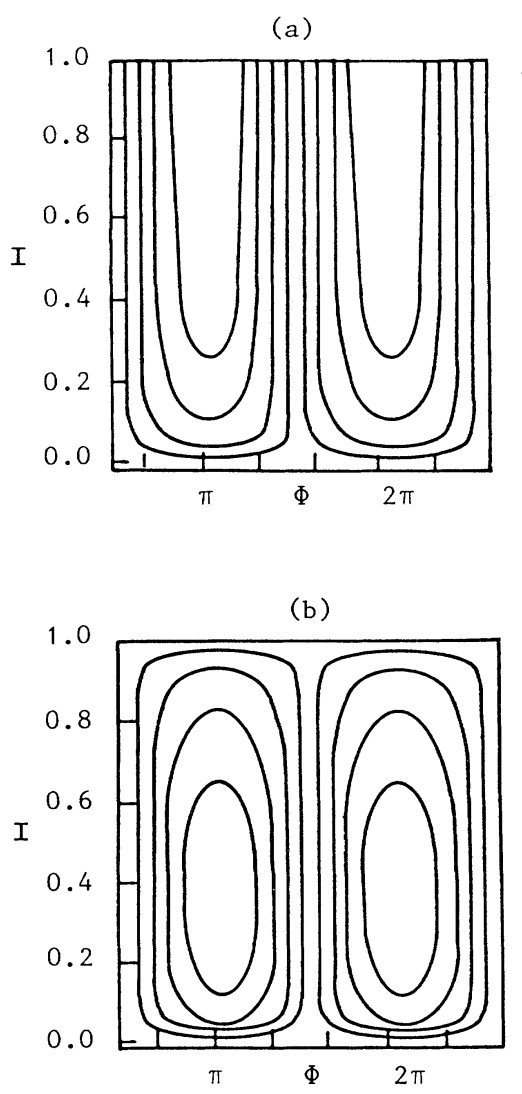

FIG. 2. Phase-space trajectories for $f=1$. In (a) wave dynamics is not included and in (b) it is. We consider $\rho=1.0$, $h=1$, and $\lambda=0.01$.

jectories in the phase space for $f=1$. It can be observed that the upwardly open curves of Fig. 1, where wave dynamics is disregarded $(\rho-I \rightarrow \rho)$, are closed in Fig. 2, where wave dynamics is taken into account. In Fig. 3 one considers the situation $f=0.8$ with wave dynamics included. There one notices that, much in accordance with our analytical estimates, relocation of the two classes of fixed points is already observable.

Interesting to mention is that the sequence of all canonical transformations applied so far allows one to obtain the following relation for the particle normalized perpendicular velocity:

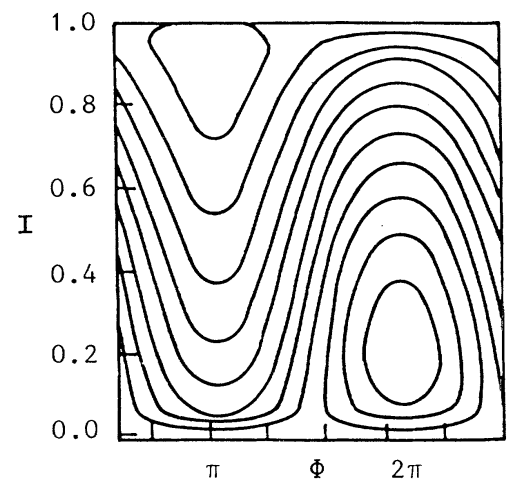

FIG. 3. The same as in Fig. 2 but for $f=0.8$.

$$
v_{\perp}^{2}=\frac{2 I}{1+2 I+\left(P_{z}+f I\right)^{2}} .
$$

For $f=1$ and $P_{z}=0$, such a relation indicates that, regardless of the form of the actual dynamics, transverse acceleration must be a bounded process, because, as already noticed in Ref. [1], the maximum of the function represented by Eq. (25) is given by $v_{\perp, \max }^{2}=0.5$. On the other hand, as we allow for smaller values of $f$, this upper bound increases; in the limit $f \ll 1$, relation (25) sets no upper bounds on $v_{\perp}$ besides the natural one $v_{\perp}^{2}<1$. The accessibility of such a kind of large velocity seems to be a relevant point for discussion. Nevertheless, it is a complicated matter and should be left for a future publication.

To summarize, we have performed an analysis of the wave-particle self-consistent dynamical interaction that takes place in a generic cyclotron-laser system. By means of a canonical procedure we have been able to determine two basic characteristics for this kind of device: (i) upper bounds for particle energization processes and (ii) the presence and location of fixed points for the overall dynamics. In specific terms, we have shown how wave dispersion and the usually discarded wave dynamics act as limiters for energization processes; in particular, it has highlighted the importance of the finite amount of electromagnetic energy for those situations where the electromagnetic wave propagates without dispersion.

This work was partially supported by Financiadora de Estudos e Projetos (FINEP) and Conselho Nacional de Desenvolvimento Científico e Tecnológico (CNPq), Brazil.
[1] S. P. Kuo and G. Schmidt, J. Appl. Phys. 58, 3646 (1985).

[2] F. B. Rizzato, Phys. Scr. 46, 173 (1992).

[3] P. Sprangle and A. T. Drobot, IEEE Trans. Microwave Theory Tech. MTT-25, 528 (1977).

[4] K. R. Chu, A. T. Drobot, H. H. Szu, and P. Sprangle, IEEE Trans. Microwave Theory Tech. MTT-28, 313 (1980).

[5] C. Chen, Phys. Fluids B 3, 2933 (1991).
[6] K. D. Pendergast, B. G. Danly, R. J. Temkin, and J. S. Wurtele, IEEE Trans. Plasma Science P-16, 122 (1988).

[7] J. K. Lee, W. D. Bard, S. C. Chiu, R. C. Davidson, and R. R. Goforth, Phys. Fluids 31, 1824 (1988).

[8] K. R. Chen, J. M. Dawson, A. T. Lin, and T. Katsouleas, Phys. Fluids B 3, 1270 (1991).

[9] F. B. Rizzato, Phys. Rev. A 41, 1629 (1990).

[10] T. M. Antonsen, Jr. and G. Laval, Phys. Fluids B 1, 1721 (1989). 